\title{
A tissue-engineered subcutaneous pancreatic cancer model for antitumor drug evaluation
}

This article was published in the following Dove Press journal:

International Journal of Nanomedicine

18 March 2013

Number of times this article has been viewed

\author{
Qingyi $\mathrm{He}^{2, *}$ \\ Xiaohui Wang,* \\ Xing Zhang ${ }^{4}$ \\ Huifang $\mathrm{Han}^{4}$ \\ Baosan $\mathrm{Han}^{5}$ \\ Jianzhong $\mathrm{Xu}^{2}$ \\ Kanglai Tang ${ }^{2}$ \\ Zhiren Fu' \\ Hao Yin ${ }^{1,4}$ \\ 'Department of Surgery, Organ \\ Transplant Center, Shanghai \\ Changzheng Hospital, Shanghai Second \\ Military Medical School, ${ }^{2}$ Department \\ of Orthopedic Surgery, Southwest \\ Hospital, Third Military Medical \\ University, Chongqing, ${ }^{3}$ Department \\ of General Surgery, Bayinguoleng \\ Mengguzizhizhou People's Hospital, \\ Korla, Xinjiang, People's Republic \\ of China; ${ }^{4}$ Department of Surgery, \\ The University of Chicago, Chicago, \\ IL, USA; ${ }^{5}$ Department of Surgery, \\ Shanghai Xinhua Hospital, Shanghai, \\ People's Republic of China \\ *These authors contributed equally \\ to this work
}

\begin{abstract}
The traditional xenograft subcutaneous pancreatic cancer model is notorious for its low incidence of tumor formation, inconsistent results for the chemotherapeutic effects of drug molecules of interest, and a poor predictive capability for the clinical efficacy of novel drugs. These drawbacks are attributed to a variety of factors, including inoculation of heterogeneous tumor cells from patients with different pathological histories, and use of poorly defined Matrige $l^{\circledR}$. In this study, we aimed to tissue-engineer a pancreatic cancer model that could readily cultivate a pancreatic tumor derived from highly homogenous $\mathrm{CD} 24^{+} \mathrm{CD} 44^{+}$pancreatic cancer stem cells delivered by a well defined electrospun scaffold of poly(glycolide-co-trimethylene carbonate) and gelatin. The scaffold supported in vitro tumorigenesis from $\mathrm{CD} 24^{+} \mathrm{CD} 44^{+}$cancer stem cells for up to 7 days without inducing apoptosis. Moreover, CD24 ${ }^{+} \mathrm{CD} 44^{+}$cancer stem cells delivered by the scaffold grew into a native-like mature pancreatic tumor within 8 weeks in vivo and exhibited accelerated tumorigenesis as well as a higher incidence of tumor formation than the traditional model. In the scaffold model, we discovered that oxaliplatin-gemcitabine (OXA-GEM), a chemotherapeutic regimen, induced tumor regression whereas gemcitabine alone only capped tumor growth. The mechanistic study attributed the superior antitumorigenic performance of OXA-GEM to its ability to induce apoptosis of CD $24^{+} \mathrm{CD} 44^{+}$cancer stem cells. Compared with the traditional model, the scaffold model demonstrated a higher incidence of tumor formation and accelerated tumor growth. Use of a tiny population of highly homogenous $\mathrm{CD} 24^{+} \mathrm{CD} 44^{+}$cancer stem cells delivered by a well defined scaffold greatly reduces the variability associated with the traditional model, which uses a heterogeneous tumor cell population and poorly defined Matrigel. The scaffold model is a robust platform for investigating the antitumorigenesis mechanism of novel chemotherapeutic drugs with a special focus on cancer stem cells.
\end{abstract}

Keywords: pancreatic cancer, cancer stem cell, electrospun scaffold, gemcitabine, oxaliplatin

\section{Introduction}

Pancreatic carcinoma remains one of deadliest cancers, with the tumor having already metastasized to other organs at the time of diagnosis in over $80 \%$ of patients, depriving patients of the option to have the tumor surgically removed. Consequently, the 5 -year survival rate for patients with pancreatic carcinoma is less than $5 \%$. It does not help that the last two decades has yielded only one clinically potent chemotherapeutic drug, gemcitabine, which can increase the patient's life for only 6 months by average. ${ }^{1}$ Despite the enormous effort invested in discovering novel chemotherapeutic drugs, the majority of molecules that seemed promising in murine models have failed in various phases of clinical trials. ${ }^{1-4}$ This disappointing outcome has prompted scientists to 
rethink the direction in which they have been traveling in the recent decades. A consensus has thus emerged that the murine tumor model must be improved to increase the efficacy and reliability of chemotherapeutic drug discovery research. ${ }^{5,6}$ The traditional subcutaneous tumor model is established by implanting a freshly resected human tumor mass on the flank in immunodeficient mice and allowing it to grow to a sizeable tumor for drug screening. This model gained wide popularity in the pharmaceutical industry because of its surgical convenience and low cost for large-scale drug screening. In contrast, the xenograft orthotopic model is surgically challenging, while genetically engineered mice are prohibitively expensive for widespread use in the pharmaceutical industry. ${ }^{7-9}$ Therefore, the subcutaneous model remains a valuable platform for evaluating the efficacy of novel chemotherapeutic drugs in both academia and in the pharmaceutical industry.

The traditional subcutaneous pancreatic tumor model suffers from a number of drawbacks. For example, only a fraction of mice that undergo subcutaneous implantation will ultimately grow a sizeable tumor mass within a few weeks. Moreover, freshly resected human pancreatic tumor is not readily available and usually needs immediate processing and implantation, which is cumbersome in experimental research. Worse still is the fact that pancreatic tumor specimens resected from human patients with different pathological backgrounds jeopardize the reproducibility of results pertaining to the efficacy of novel chemotherapeutic drugs. Being aware of these limitations, we have strived to tissueengineer a novel subcutaneous pancreatic cancer model by using a highly homogeneous population of pancreatic cancer stem cells and a well defined electrospun scaffold. Use of cancer stem cells gives cancer scientists a large time window to build a model at their convenience and greatly reduces the pathological variability of implanted cancer cells associated with the traditional model.

Previous research has shown that as few as hundreds of highly tumorigenic cancer stem cells can grow into a sizeable tumor mass in vivo and that these cancer stem cells can be passaged in vitro without differentiating into various mature cancer cells. ${ }^{10-15}$ Based on previous research, we used $\mathrm{CD} 24^{+} \mathrm{CD} 44^{+}$cancer stem cells from a resected human pancreatic tumor specimen to grow pancreatic tumor tissue in our novel model. ${ }^{10,11,13}$ In the traditional cancer model, delivering a tiny population of cancer stem cells sometimes requires a supporting matrix, most commonly Matrige ${ }^{\circledR}(\mathrm{BD}$ Biosciences, Franklin Lakes, NJ, USA) to provide cancer stem cells with a microenvironment in which to survive and proliferate after implantation. However, Matrigel is a poorly defined matrix with noticeable variation in chemical composition from batch to batch, thus inevitably reducing the reproducibility of drug screening results. Tissue-engineering scientists have extensively explored how to build a well defined scaffold from synthetic polymers and proteins to promote tissue growth and/or regeneration, which offers a powerful tool for cancer research. ${ }^{16-26}$ Consequently, based on our previous research, we tissue-engineered a highly porous scaffold by electrospinning poly(glycolide-co-trimethylene carbonate) (PGA-TMC) and gelatin to provide the implanted $\mathrm{CD} 24^{+} \mathrm{CD} 44^{+}$cancer stem cells with a favorable and well defined microenvironment in which to survive and proliferate in vivo, ${ }^{21}$ which would eliminate the unfathomable influence exerted by Matrigel. Thereafter, we mechanistically investigated the increased antitumorigenic effect of an oxaliplatin-gemcitabine (OXA-GEM) chemotherapeutic regimen compared with gemcitabine alone by taking advantage of this scaffold model.

\section{Materials and methods \\ Fabrication and morphological characterization of scaffold}

The scaffold was electrospun as previously described. ${ }^{21}$ Briefly, PGA-TMC (Advanced Inventory Management, Mokena, IL, USA) and porcine gelatin A (Sigma-Aldrich, St Louis, MO, USA) were dissolved in 1,1,1,3,3,3-hexafluoro-2propanol (Sigma-Aldrich) to achieve a total concentration of $12 \%(\mathrm{w} / \mathrm{v})$ and a weight ratio of 5:1. After being loaded into a 27-gauge needle, the solution was subjected to a voltage of $30 \mathrm{kV}$ (M826, Gamma High-Voltage Research, Ormond Beach, FL, USA) for electrospinning. The solution was fed at a rate of $3 \mathrm{~mL}$ per hour with the collector distanced at $25 \mathrm{~cm}$ from the needle. After electrospinning, the scaffold was desiccated in vacuum for at least 24 hours. An electrospun scaffold sample measuring $1 \mathrm{~cm} \times 1 \mathrm{~cm}$ was sputter-coated with gold, and scanning electron microscopy was used to capture images for morphological analysis of the fibers.

\section{Collection of CD24+CD44+ cancer stem cells from resected human pancreatic tumors}

Pancreatic tumors were resected from human patients at Shanghai Changzheng Hospital. The resected tumor tissues were completely disassociated with scissors and blades in a sterile environment. The disassociated tissues were then incubated with ultrapure collagenase IV (Worthington Biochemicals, Freehold, NJ, USA) in medium 199 (200 units 
of collagenase per $\mathrm{mL}$ ) at $37^{\circ} \mathrm{C}$ for 4 hours to obtain a singlecell suspension. During incubation, the cells were further agitated with a pipette every 30 minutes. After incubation, the suspension was filtered through a $40 \mu \mathrm{m}$ nylon mesh, washed with Hank's Balanced Salt Solution with 20\% fetal bovine serum, and then rinsed at least three times with Hank's Balanced Salt Solution. The CD $24^{+} \mathrm{CD} 44^{+}$cancer stem cells were sorted by fluorescent-activated cell sorting (BD LSRII, BD Biosciences, San Jose, CA, USA) using antibodies of antihuman CD24 and antihuman CD44 (Pharmingen, Franklin Lakes, NJ, USA). The sorting was repeated until purification reached over $90 \%$. The sorted CD $24^{+} \mathrm{CD} 44^{+}$ cancer stem cells were cultured as previous described, and cells between passages 2 and 4 were used. ${ }^{27}$ Written informed consent was obtained from patients prior to resection of the pancreatic tumor specimens.

\section{In vitro analysis of viability and apoptosis in $\mathrm{CD} 24^{+} \mathrm{CD} 44^{+}$cancer stem cells on scaffold}

Circular scaffolds (diameter $6 \mathrm{~mm}$ ) were sterilized in $70 \%$ ethanol for 15 minutes followed by an extensive rinse in sterile phosphate-buffered solution. The $\mathrm{CD} 24^{+} \mathrm{CD} 44^{+}$cancer stem cells were seeded on the scaffold (1000 cells/scaffold) and cultured at $37^{\circ} \mathrm{C}$ and $5 \% \mathrm{CO}_{2}$ for up to 7 days to allow tumorigenesis. Viability and apoptosis in the pancreatic tumor cells were measured using a 3-(4,5-dimethylthiazol2-yl)-2,5-diphenyltetrazolium bromide (MTT) and CaspaseGlo $^{\circledR}$ 3/7 assay kit, respectively, as per the manufacturer's protocols (Promega, Madison, WI, USA) on days 1 and 7. $\mathrm{CD} 24^{+} \mathrm{CD} 44^{+}$cancer stem cells cultured in a 96-well tissue culture plate (1000 cells/well) were used as the control.

\section{Surgical creation of tissue-engineered subcutaneous (scaffold) model}

$\mathrm{CD} 24^{+} \mathrm{CD} 44^{+}$cancer stem cells were seeded on sterilized scaffolds (diameter $3 \mathrm{~mm}, 5000$ cells/scaffold) and incubated at $37^{\circ} \mathrm{C}$ and $5 \% \mathrm{CO}_{2}$ overnight. The cancer stem cell/ scaffold construct was then implanted subdermally on the flank of each female mouse (BALB/c nu/nu, 4-6 weeks old, Shanghai Laboratory Animal Co, Shanghai, People's Republic of China). In the control group, $\mathrm{CD} 24^{+} \mathrm{CD} 44^{+}$cancer stem cells were freely injected subdermally on the flank of each mouse (5000 cells/mouse, traditional model). The mice were anesthetized by inhalation of vaporized isoflurane during surgery. All mice were sacrificed at week 8 after surgery. All the animal studies were approved by Shanghai Changzheng Hospital.

\section{Measurements of in vivo pancreatic tumor growth}

The tumor site was photographed biweekly and the volume was calculated as previously described (tumor volume $\left.=\left[\mathrm{L} \times \mathrm{W}^{2}\right] / 2.28\right)$. After sacrifice, the tumor mass was retrieved and its weight was measured on a toploading digital balance.

\section{Phenotypic analysis of tumor cells by immunohistochemistry}

After sacrifice, the pancreatic tumor masses retrieved from the scaffold model were fixed in 10\% formalin for 48 hours, then embedded in paraffin, sliced into $4 \mu \mathrm{m}$ thick sections, and serially collected on charged glass slides. Carbohydrate antigen 19-9 (Abcam, Cambridge, MA, USA), epidermal growth factor (Abcam), and MIB-1 (LifeSpan Biosciences, Seattle, WA, USA) were used to characterize the phenotype of the tumor cells using immunohistochemistry staining following the manufacturers' protocols. A freshly resected human pancreatic tumor specimen was used as the positive control.

\section{Comparative study of antitumorigenic effect of OXA-GEM and gemcitabine in scaffold model}

Oxaliplatin and gemcitabine were obtained from Shanghai Changzheng Hospital. The scaffold model was established as described above. At week 5 following surgery, the mice were randomly assigned to an OXA-GEM group, a gemcitabine group, or a phosphate-buffered solution (control) group. Oxaliplatin $4 \mathrm{mg} / \mathrm{kg}$ was first given to each mouse via intraperitoneal injection, followed immediately by gemcitabine $50 \mathrm{mg} / \mathrm{kg}$ in the OXA-GEM group. Gemcitabine $50 \mathrm{mg} / \mathrm{kg}$ and sterile phosphate-buffered solution were given by intraperitoneal injection to mice in the gemcitabine group and the control group, respectively. All drugs were administered weekly for a total of 4 weeks. The dose of each drug was determined from previous clinical research. ${ }^{29}$ All mice were sacrificed after 4 weeks of drug administration. Tumor volume and weight were measured as described above. Retrieved tumor samples were prepared for flow cytometric analysis of $\mathrm{CD} 22^{+} \mathrm{CD} 44^{+}$cancer stem cells, again as described above.

\section{Mechanistic study of OXA-GEM and gemcitabine in pancreatic-derived CD $24^{+}$CD $44^{+}$cancer stem cells}

$\mathrm{CD} 24^{+} \mathrm{CD} 44^{+}$cancer stem cells were seeded on sterilized scaffolds (diameter 6 mm, 1000 cells/scaffold) and cultured 
at $37^{\circ} \mathrm{C}$ and $5 \% \mathrm{CO}_{2}$ for 5 days without drug administration. The cells were then cultured in medium supplemented with OXA-GEM (oxaliplatin $8 \mathrm{nM}$ and gemcitabine $100 \mathrm{nM}$ ), gemcitabine (100 nM), or phosphate-buffered solution for 4 days. MTT and Caspase-Glo ${ }^{\circledR}$ 3/7 assay kits were used to measure viability and apoptosis of the tumor cells on day 5 (before drug treatment) and day 9 (after drug treatment).

\section{Data analysis}

All immunohistochemistry slides were scanned using a CRi Pannoramic Scan whole slide scanner, and the images were captured and analyzed using the Pannoramic viewer (3DHistech, Budapest, Hungary) and ImageJ. FlowJo (Tree Star Inc, Ashland, OR, USA) was used in the flow cytometric analysis. The Student's $t$-test and analysis of variance with the Tukey test were used where applicable $(\alpha=0.05)$. All results are presented as the mean \pm standard deviation.

\section{Results and discussion Morphological characterization of electrospun scaffold}

The electrospun scaffold was composed of randomly distributed nonwoven micro/nanofibers, featuring a highly porous structure that morphologically mimicked the native extracellular matrix (Figure 1A). The scaffold also showed a highly homogeneous fiber morphology, with the majority of fibers having a diameter of 4-6 $\mu \mathrm{m}$ (Figure 1B). It should be noted that each individual fiber in the scaffold was a composite of PGA-TMC and gelatin. In tissue engineering research, a scaffold is intended to provide a temporal physical environment for therapeutic regeneration of tissue. To that end, it is believed that a functional scaffold should be morphologically analogous to the native extracellular matrix which supports adhesion, migration, and proliferation of cells. In addition, previous research has demonstrated that the functional output of the scaffold could be further enhanced if biological domains in the native extracellular matrix could be present in the scaffold. ${ }^{20,25,26}$ Meanwhile, the scaffold is expected to degrade in a coordinated fashion as cells produce their native extracellular matrix proteins. In the recent decades, scaffolds constructed from a combination of proteins, polymers, and inorganic materials (eg, hydroxyapatite) have been extensively investigated to create a microenvironment that mimics the native extracellular matrix. ${ }^{30}$ These engineered scaffolds have been used to regenerate a great variety of tissues, including bones, blood vessels, and heart valves. Previous research has shown that tissue-engineered scaffolds could play a pivotal role in initiating and regulating therapeutic regeneration of tissue. ${ }^{16-19,25,26}$ For example, biochemical cues like growth factors incorporated into the scaffold can exert a regulatory effect on important cellular events, including metastasis of cancer cells and differentiation of stem cells. Based on previous research, we used PGA-TMC to confer structural integrity to the scaffold, so that seeded cells could readily adhere and selforganize into a functional tissue. ${ }^{21} \mathrm{PGA}-\mathrm{TMC}$ is a degradable synthetic polymer with no cytotoxicity in vivo, and is widely used for biomedical applications, including surgical sutures and tissue-engineered scaffolds. ${ }^{31,32}$ To enhance its biological performance, we incorporated gelatin to facilitate integrinmediated cell adhesion. Our previous research confirmed that the PGA-TMC/gelatin scaffold held promise for tissue regeneration because it possesses an extracellular matrix-like microstructure as well as tunable degradation. ${ }^{21}$

\section{In vitro tumorigenesis and apoptosis of $\mathrm{CD} 24^{+} \mathrm{CD} 44^{+}$cancer stem cells}

To determine if the scaffold could promote pancreatic tumorigenesis from $\mathrm{CD} 24^{+} \mathrm{CD} 44^{+}$cancer stem cells, we cultured
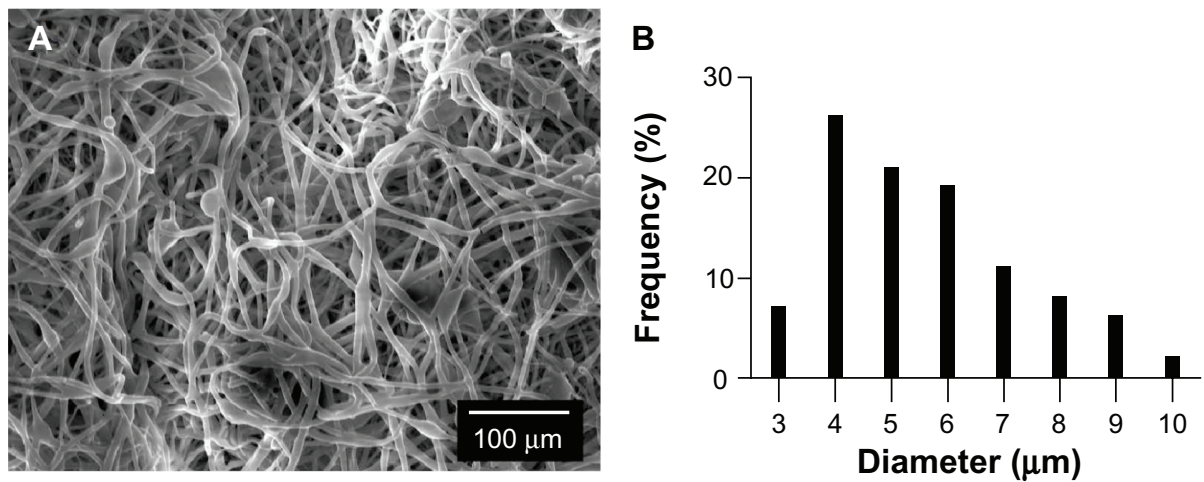

Figure I (A) Scanning electron micrograph of an electrospun scaffold and (B) fiber diameter distribution.

Notes: The micrograph shows a highly porous microstructure analogous to the protein network in a native extracellular matrix. Measurement of fiber diameter showed that the fibers were highly homogeneous, with most having a diameter of 4-6 $\mu \mathrm{m}$. 
these cells on the scaffold in vitro for up to 7 days and measured the viability and apoptosis of tumor cells on days 1 and 7. The MTT assay showed no difference with regard to viability of CD $24^{+} \mathrm{CD} 44^{+}$cancer stem cell-derived tumor cells on the scaffold and on a tissue culture plate on day 1. However, $\mathrm{CD} 24^{+} \mathrm{CD} 44^{+}$cancer stem cell-derived tumor cells showed higher viability than their counterparts on the tissue culture plate on day 7 , suggesting that the scaffold promoted a more favorable biophysical environment for $\mathrm{CD} 24^{+} \mathrm{CD} 44^{+}$cancer stem cells to grow into a mature pancreatic tumor (Figure 2). Neither the scaffold nor the tissue culture plate induced the apoptosis of $\mathrm{CD} 24^{+} \mathrm{CD} 44^{+}$cancer stem cells or tumor cells derived from them on days 1 and 7 (Figure 3), indicating a lack of cytotoxic effect from the scaffold materials.

It is long established that the microenvironment has a profound influence on proliferation of cancer cells. ${ }^{16-20,33}$ For example, it was shown that cancer cells cultured in a threedimensional matrix had enhanced angiogenesis and greater resistance to chemotherapeutic drugs than those cultured in a two-dimensional matrix..$^{33}$ Therefore, the ability of $\mathrm{CD} 24^{+} \mathrm{CD} 44^{+}$cancer stem cells to adhere and proliferate readily on the scaffold determines the final success of this tissueengineered pancreatic cancer model. Our results confirmed that $\mathrm{CD} 24^{+} \mathrm{CD} 44^{+}$cancer stem cells cultured on the scaffold had a stronger proliferative capacity than those cultured on a tissue culture plate, as evidenced by increased viability on day 7 without provocation of apoptosis. It must be noted that $\mathrm{CD} 24^{+} \mathrm{CD} 44^{+}$cancer stem cells might have grown and differentiated into a heterogeneous population of pancreatic cancer cells on the scaffold during the 7-day period, because pancreatic cancer stem cells would start to differentiate after they adhered to a substrate. Such differentiation is desirable,

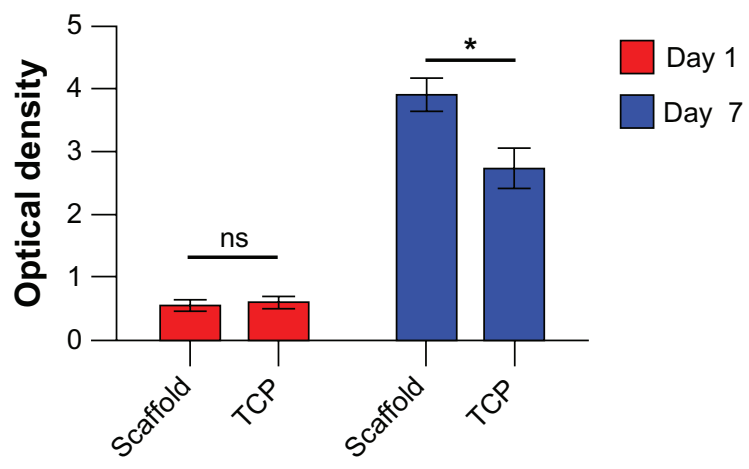

Figure 2 Analysis of viability of $\mathrm{CD} 24^{+} \mathrm{CD} 44^{+}$cancer stem cell-derived pancreatic tumor cells on the scaffold showing no difference on day $I$.

Notes: However, cells on the scaffold outperformed controls on the tissue culture plate by day 7 , suggesting that the scaffold provided a more favorable microenvironment than the tissue culture plate for cancer stem cells to grow into pancreatic tumors. A star denotes a difference between the groups connected by a hanging bar $(n=3)$. Abbreviation: TCP, tumor control probability; ns, not significant.

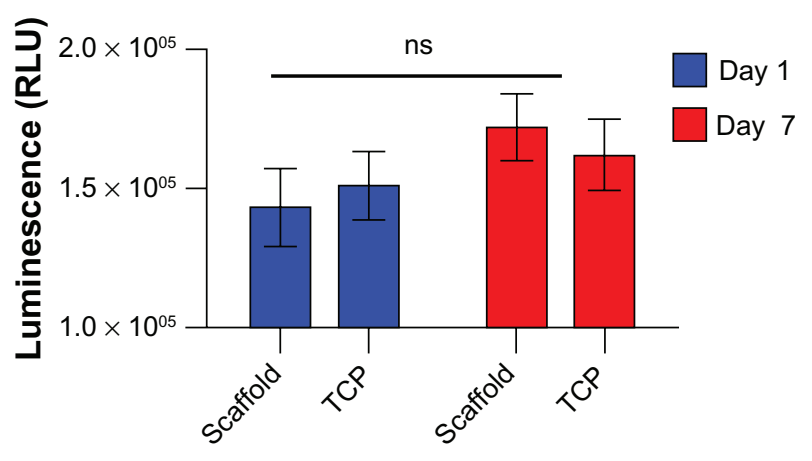

Figure 3 Analysis of apoptosis in CD24 $4^{+} \mathrm{CD} 44^{+}$cancer stem cell-derived pancreatic tumor cells on the scaffold.

Notes: Neither the scaffold nor the tissue culture plate caused significant apoptosis, indicating that the scaffolding materials were not cytotoxic $(n=3)$. Moreover, no increase in apoptosis was observed in either group during days 1 to 7 , suggesting that the degrading scaffold materials were not cytotoxic.

Abbreviation: TCP, tumor control probability; ns, not significant.

given that a fully-fledged pancreatic tumor is wanted, rather than a large population of $\mathrm{CD} 24^{+} \mathrm{CD} 44^{+}$cancer stem cells.

\section{In vivo tumorigenesis and phenotypic characterization of pancreatic tumor originating from $\mathrm{CD} 24^{+} \mathrm{CD} 44^{+}$cancer stem cells}

After being implanted subdermally in mice for 8 weeks, the $\mathrm{CD} 24^{+} \mathrm{CD} 44^{+}$cancer stem cell-derived pancreatic tumor in the scaffold model outgrew its counterpart in the traditional model by $44.48 \%$ in volume and $91.58 \%$ in weight (Figure 4). By week 6 after surgery, the tumor volume in the scaffold model reached $1156 \pm 144 \mathrm{~mm}^{3}$, but reached only $780 \pm 105 \mathrm{~mm}^{3}$ in the traditional model, and this difference was statistically significant. By week 8 , the volume in the scaffold model increased further to $1478 \pm 162 \mathrm{~mm}^{3}$ compared with just $1023 \pm 149 \mathrm{~mm}^{3}$ in the traditional model. The tumor weight in the scaffold model and traditional model was $182 \pm 32 \mathrm{mg}$ and $95 \pm 27 \mathrm{mg}$, respectively, by week 8 after surgery. In addition, in the scaffold model group, eight of ten mice showed growth of a CD24+CD $44^{+}$cancer stem cell-derived tumor, representing an $80 \%$ success rate, while the success rate in the traditional model was only $50 \%$ (five of ten). Phenotypic characterization by immunohistochemistry showed that $\mathrm{CD} 24^{+} \mathrm{CD} 44^{+}$cancer stem cell-derived tumor cells in the scaffold model readily expressed pancreatic cancer markers, including carbohydrate antigen 19-9, epidermal growth factor, and MIB-1, confirming that the scaffold model could reliably cultivate a mature native-like pancreatic tumor (Figure 5).

One of the technical challenges in cancer research is that the incidence of tumor formation in a subcutaneous model is 
A

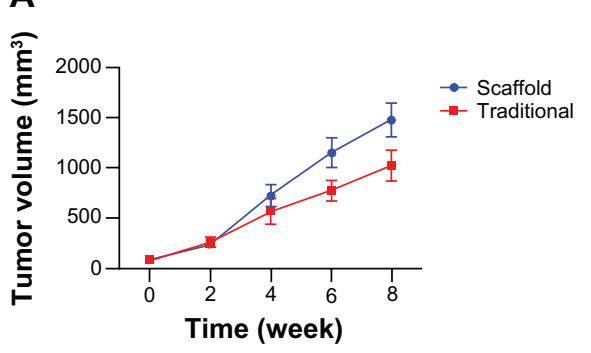

B

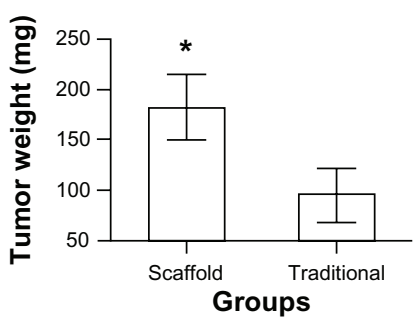

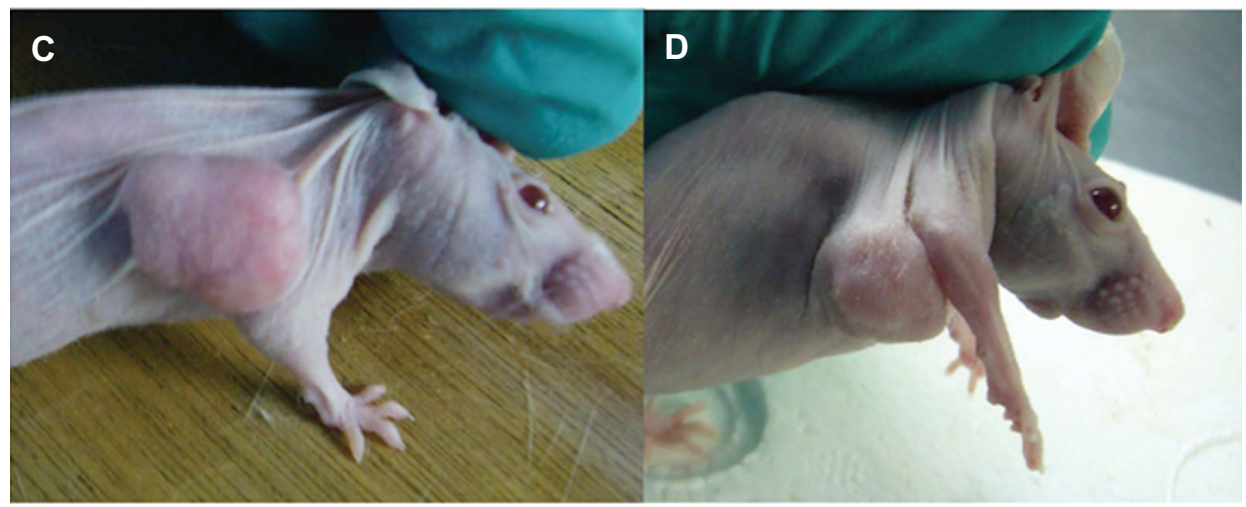

Figure $4(\mathrm{~A})$ Volume of tumors originating from implanted $C D 24^{+} \mathrm{CD} 44^{+}$cancer stem cells and $(\mathrm{B})$ weight of tumors from each group, along with representative photographs of pancreatic tumors from the scaffold model (C) and the traditional model (D).

Notes: While both models showed steady tumorigenesis through to week 8 , the tumor volume in the scaffold model was greater than in the traditional model by week 6 , and this growth pattern continued until week 8 . Tumor weight in the scaffold model was also higher than in the traditional model. These results confirm that the scaffold promoted tumorigenesis, which could be attributed to enhanced survival during implantation. $n=8$ in the scaffold model; $n=5$ in the traditional model.

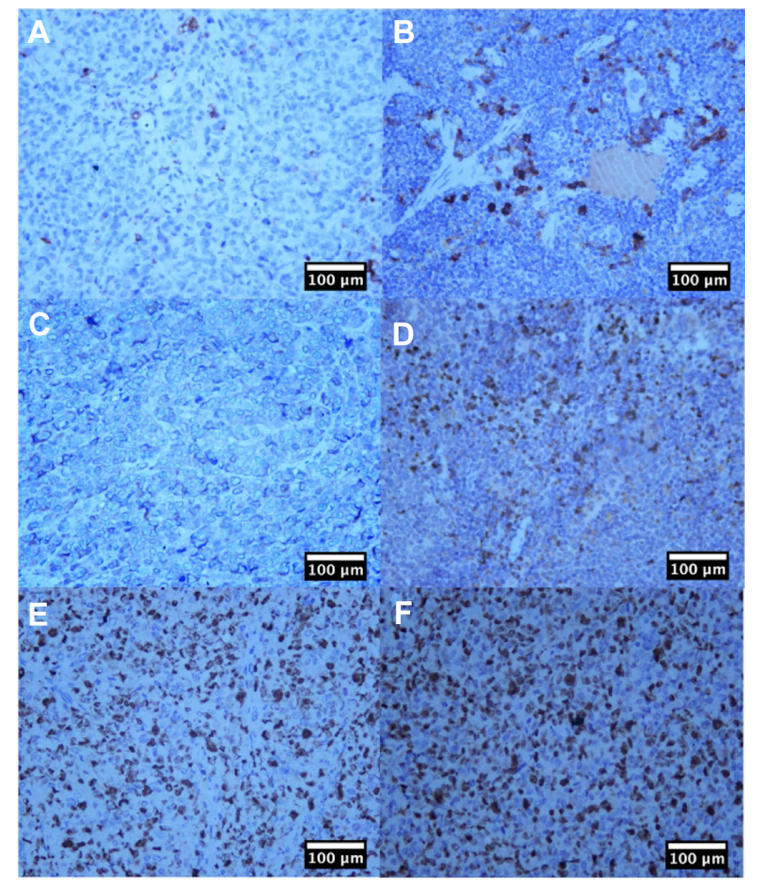

Figure 5 Immunohistochemistry staining of CA-199 (A and B), epidermal growth factor (C and D) and MIB-I (E and F).

Notes: The left column shows samples from freshly resected human pancreatic tumors (positive control) and the right column shows samples from pancreatic tumors retrieved from mice in the scaffold model. Tumor samples retrieved from the scaffold model showed expression of all three pancreatic cancer markers, confirming that tumors derived from $\mathrm{CD} 24^{+} \mathrm{CD} 44^{+}$cancer stem cells were biologically equivalent to their native counterparts. low and unpredictable, which could be attributed to several factors. First of all, previous cancer research has shown that only a small fraction of a large heterogeneous population of cancer cells in a tumor is highly tumorigenic, and this is believed to be the driving force in tumor formation. ${ }^{10-12,14}$ In the traditional subcutaneous model, a sizeable resected human pancreatic tumor mass was implanted and allowed to grow in vivo for a few weeks. However, the availability of cancer stem cells in the resected mass varies greatly, leading to a volatile reliability of this model. Further, a favorable microenvironment is absent in the subcutaneous space in the traditional model, depriving the implanted cells of a good opportunity to adhere and proliferate. Finally, the highly heterogeneous nature of the tumor mass in the traditional model greatly compounds interpretation of the variability observed in chemotherapeutic drug evaluation.

To address these concerns, we hypothesized that we could engineer a native-like pancreatic tumor from a tiny population of highly homogenous and tumorigenic $\mathrm{CD} 24^{+} \mathrm{CD} 44^{+}$ cancer stem cells, which could both increase the likelihood of tumor formation and yield reliable results in drug evaluation studies. In the traditional subcutaneous model, Matrigel is sometimes used when delivering a small population of cancer cells. The fact that Matrigel is a poorly defined material 


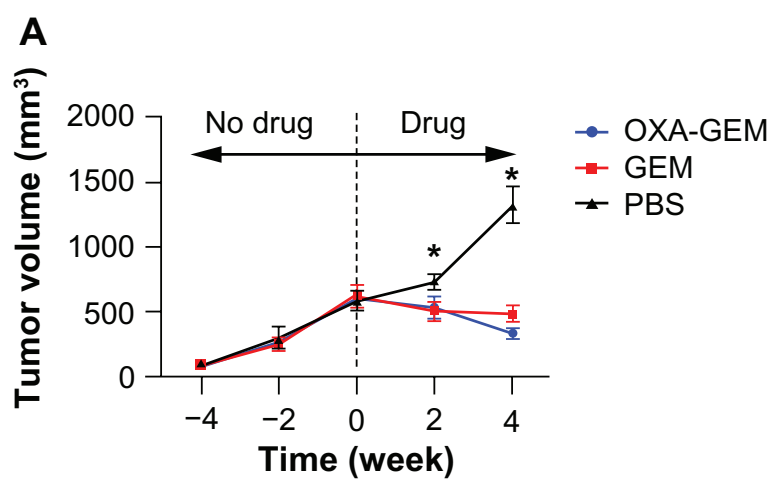

B

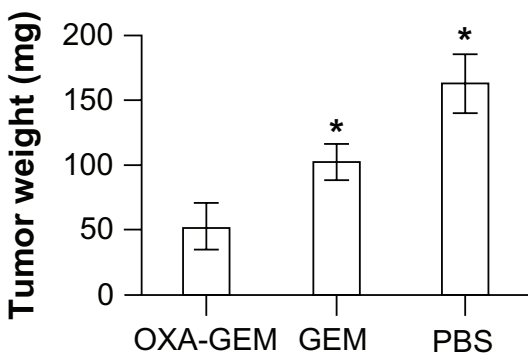

Figure 6 (A) Volume and (B) weight of tumors in each group.

Notes: After 2 weeks of drug administration, tumor volumes in the OXA-GEM and gemcitabine groups were both smaller than those in the control group, confirming that OXA-GEM and gemcitabine could arrest tumorigenesis. However, after 4 weeks of drug administration, the tumor volume in the OXA-GEM group was lower than that in the gemcitabine group, and the tumor volumes in both groups were significantly smaller than the tumor volume in the control group. Tumor volume in the OXA-GEM group showed a decrease after 4 weeks of drug administration while tumor volume in the gemcitabine group remained unchanged. Correspondingly, after 4 weeks of drug administration, tumor weight in the OXA-GEM group was lower than that in the gemcitabine group, with that in the control group lagging behind ( $\mathrm{n}=8$ in the OXA-GEM group; $n=9$ in the gemcitabine group; $n=8$ in the control group). A star denotes a statistically significant difference between the groups.

Abbreviations: OXA-GEM, oxaliplatin-gemcitabine; GEM, gemcitabine; PBS, phosphate-buffered solution.

introduces unknown factors into the traditional pancreatic cancer model, and thus impedes identification of the reason for inconsistent drug efficacy results. In light of these challenges, a well defined matrix is of great significance. To boost the survival rate of $\mathrm{CD} 24^{+} \mathrm{CD} 44^{+}$cancer stem cells, we tissue-engineered an extracellular matrix-like scaffold to afford a favorable microenvironment in which the cells could adhere, migrate, and proliferate, without provoking massive apoptosis. Our results confirmed an increased incidence of tumor formation and accelerated tumor growth within an 8-week period.

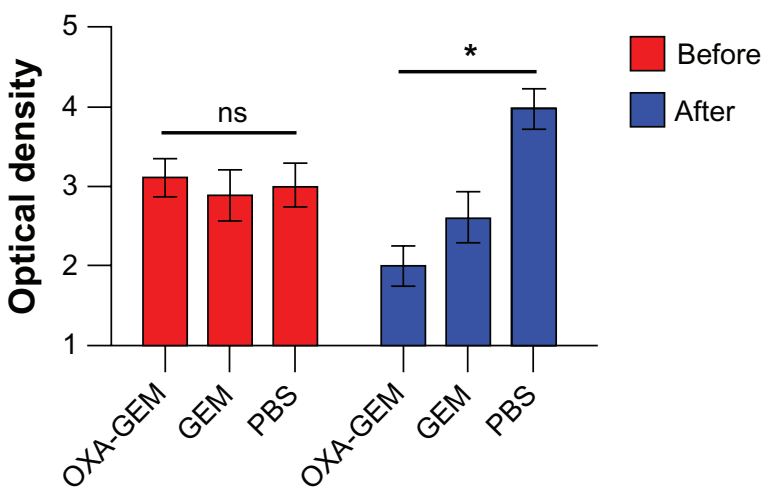

Figure 7 Viability analysis of $\mathrm{CD} 24^{+} \mathrm{CD} 44^{+}$cancer stem cell-derived pancreatic tumor cells before and after drug administration.

Notes: No difference was observed between the treatment groups before drug administration. However, after 4 days of drug treatment, viability in the OXA-GEM group was lower than in the gemcitabine group, with the control group showing the highest viability. During the 4-day treatment period, the viability of CD24+CD44+ cancer stem cell-derived pancreatic tumor cells decreased in the OXA-GEM group but remained unchanged in the gemcitabine group. Viability of $C D 24^{+} \mathrm{CD} 44^{+}$cancer stem cell-derived pancreatic tumor cells in the control group increased during the same time period ( $n=3$ in each treatment group). A star denotes a statistically significant difference between groups.

Abbreviations: OXA-GEM, oxaliplatin-gemcitabine; GEM, gemcitabine; PBS, phosphate-buffered solution; ns, not significant.

\section{Antitumorigenic effects of OXA-GEM and gemcitabine on pancreatic tumor derived from $\mathrm{CD} 24^{+} \mathrm{CD} 44^{+}$cancer stem cells in scaffold model}

After establishing that our scaffold model was superior to the traditional one, we took advantage of this to compare the antitumorigenic capability of oxaliplatin \pm gemcitabine, two prominent chemotherapeutic drugs for pancreatic cancer. After 2 weeks of drug administration, the tumor volume in the OXA-GEM and gemcitabine groups was significantly smaller than that in the control group. The tumor volume by week 2 after drug administration was $527 \pm 88 \mathrm{~mm}^{3}$ in the OXA-GEM group, $503 \pm 69 \mathrm{~mm}^{3}$ in the gemcitabine group, and $731 \pm 63 \mathrm{~mm}^{3}$ in the control group, with no difference between the OXA-GEM and gemcitabine groups. However, by week 4 after drug administration, the mean tumor volume was $339 \pm 45 \mathrm{~mm}^{3}$ in the OXA-GEM group, $489 \pm 61 \mathrm{~mm}^{3}$ in the gemcitabine group, and $1321 \pm 142 \mathrm{~mm}^{3}$ in the control group. The mean tumor volume in the OXA-GEM was significantly smaller than that in the gemcitabine and control groups by week 4 after drug administration. Also, the mean tumor volume in the OXA-GEM group decreased from $592 \pm 78 \mathrm{~mm}^{3}$ on week 0 to $339 \pm 45 \mathrm{~mm}^{3}$ by week 4 , while the gemcitabine group saw no decrease, indicating that OXA-GEM was a stronger chemotherapeutic regimen for pancreatic cancer (Figure 6A). After 4 weeks of drug administration, the mean tumor weight was $52 \pm 18 \mathrm{mg}$ in the OXA-GEM group, $102 \pm 14 \mathrm{mg}$ in the gemcitabine group, and $163 \pm 23 \mathrm{mg}$ in the control group (Figure 6B).

Gemcitabine has been the gold standard chemotherapeutic drug in pancreatic cancer for 20 years, but clinical research has 
A

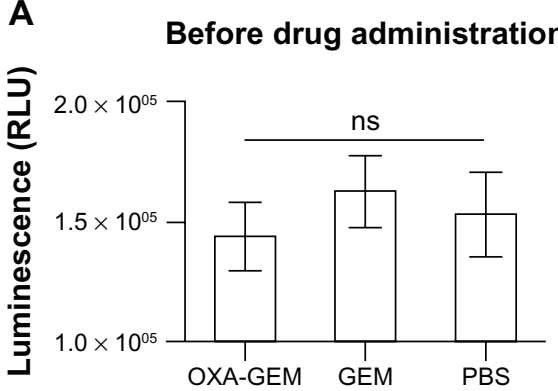

B

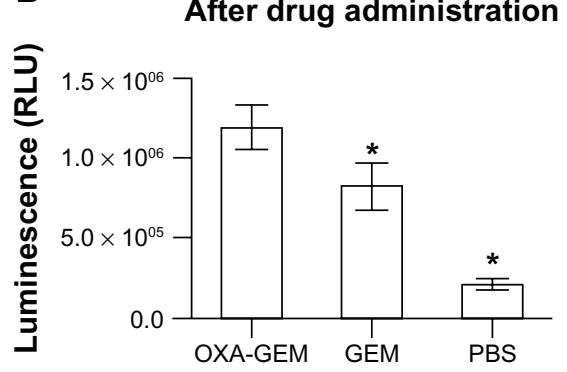

Figure 8 Analysis of apoptosis in CD24+CD44+ cancer stem cell-derived pancreatic tumor cells before (A) and after (B) drug administration. Notes: No difference was observed between the treatment groups before drug administration. However, after 4 days of drug treatment, the OXA-GEM group suffered the greatest apoptotic insult, followed by the gemcitabine group. No increase in apoptosis was observed in the control group in this time period ( $\mathrm{n}=3$ in each treatment group). A star denotes a statistically significant difference between groups.

Abbreviations: OXA-GEM, oxaliplatin-gemcitabine; GEM, gemcitabine; PBS, phosphate-buffered solution.

shown that it can only extend the patient's life by an average of roughly 6 months. ${ }^{1}$ The lackluster performance of gemcitabine prompted cancer scientists to find a better alternative, and a variety of chemotherapeutic regimens have been explored. ${ }^{1-4,29}$ Unfortunately, no breakthrough has been made, because the chemotherapeutic regimens discovered in animal models failed in various phases of clinical trials. Based on previous cancer research, we explored in this study whether an OXA-GEM regimen was superior to gemcitabine alone for pancreatic cancer. Our results showed that both OXA-GEM and gemcitabine could arrest tumorigenesis in vivo after 2 weeks, but only OXA-GEM was able to induce tumor regression after 4 weeks. These results confirm that OXA-GEM was a superior chemotherapeutic regimen for pancreatic cancer.

\section{Mechanistic study of antitumorigenic effect of OXA-GEM and gemcitabine in the scaffold model}

After confirming that OXA-GEM was superior, we proceeded to investigate the underlying mechanism for its increased antitumorigenic effect. We hypothesized that OXA-GEM had a stronger ability to eliminate $\mathrm{CD} 24^{+} \mathrm{CD} 44^{+}$cancer stem cells in pancreatic tumors, because previous findings suggested that $\mathrm{CD} 24^{+}$and $\mathrm{CD} 44^{+}$cells could be resistant to gemcitabine alone. ${ }^{34-36}$ To test our hypothesis, we first investigated whether OXA-GEM could reduce the viability of CD $24^{+} \mathrm{CD} 44^{+}$cancer stem cells more than gemcitabine alone. Our in vitro results confirmed that OXA-GEM reduced the viability of CD $24^{+} \mathrm{CD} 44^{+}$ cancer stem cells by $30 \%$ compared with gemcitabine alone within a 4-day period (Figure 7). Correspondingly, after 4 days of drug administration, the in vitro assay showed that apoptosis was $45 \%$ higher in OXA-GEM-treated CD $24^{+} \mathrm{CD} 44^{+}$ cancer stem cells than that in cells treated by gemcitabine alone (Figure 8). In addition, we measured the percentage of $\mathrm{CD} 24^{+} \mathrm{CD} 44^{+}$cancer stem cells in retrieved pancreatic tumors after 4 weeks of drug administration. The flow cytometric analysis showed that the frequency of $\mathrm{CD} 24^{+} \mathrm{CD} 44^{+}$cancer stem cells was $1.65 \% \pm 0.23 \%$ in the OXA-GEM group, $2.35 \% \pm 0.19 \%$ in the gemcitabine group, and $2.61 \% \pm 0.28 \%$ in the control group. The frequency of $\mathrm{CD} 24^{+} \mathrm{CD} 44^{+}$cancer
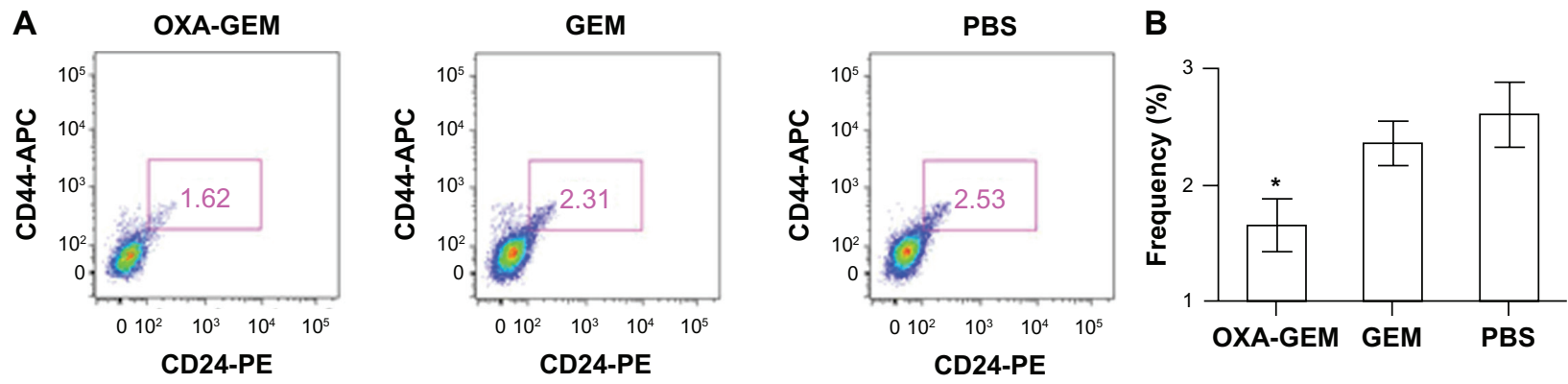

Figure 9 Frequency of $C D 24^{+} \mathrm{CD} 44^{+}$cancer stem cells after drug administration. (A) Representative flow cytometric charts of CD24+CD44 cancer stem cells after 4 weeks of drug administration. (B) Statistical analysis of the frequency of CD $24^{+} \mathrm{CD} 44^{+}$cancer stem cells in each treatment group.

Notes: OXA-GEM significantly reduced the CD24+CD44+ cancer stem cell population in pancreatic tumor tissue, depriving it of the driving force of tumorigenesis, which could account for the tumor regression observed in this group. ( $n=8$ in the OXA-GEM group; $n=9$ in the GEM group; $n=8$ in the phosphate-buffered solution group). A star denotes a statistically significant difference between groups.

Abbreviations: OXA-GEM, oxaliplatin-gemcitabine; GEM, gemcitabine; PBS, phosphate-buffered solution. 
stem cells in the OXA-GEM group was significantly lower than in the other two groups, while no difference was observed between the gemcitabine and control groups (Figure 9). Previous cancer research has demonstrated that pancreatic cancer stem cells, including CD24+ and CD $44^{+}$cells, can acquire resistance to gemcitabine during chemotherapy. ${ }^{37}$ Therefore, we complemented gemcitabine with oxaliplatin, another potent chemotherapeutic drug used for colorectal cancer, to boost the antitumorigenic capacity of $\mathrm{CD} 24^{+} \mathrm{CD} 44^{+}$cancer stem cells in pancreatic tumors. Our results confirm that addition of oxaliplatin can improve the antitumorigenic effect, which could be attributed to reduced viability and apoptosis of $\mathrm{CD} 24^{+} \mathrm{CD} 44^{+}$ cancer stem cells. It is noteworthy that OXA-GEM retained its superior antitumorigenic capacity in $\mathrm{CD} 24^{+} \mathrm{CD} 44^{+}$cancer stem cells in vivo, while gemcitabine failed to have such an antitumorigenic effect in these cells.

\section{Conclusion}

In this study, we successfully tissue-engineered a subcutaneous pancreatic cancer model from a small population of highly homogeneous and tumorigenic $\mathrm{CD} 24^{+} \mathrm{CD} 44^{+}$cancer stem cells using a well defined electrospun PGA-TMC/gelatin scaffold. This model is superior to the traditional subcutaneous model because it has a higher tumor formation rate and accelerated tumor growth in vivo, and reduces the biochemical complexity typically associated with commercial Matrigel. Further, this model is now demonstrated to be a powerful platform for mechanistic investigation of the antitumorigenic capability of chemotherapeutic regimens for pancreatic cancer.

\section{Acknowledgment}

The authors acknowledge financial support received from China's National Science Foundation (30300357, 30801107) and the Ministry of Education of Shanghai Municipal Government (09YZ84).

\section{Disclosure}

The authors report no conflicts of interest in this work.

\section{References}

1. Conroy T, Desseigne F, Ychou M, et al. FOLFIRINOX versus gemcitabine for metastatic pancreatic cancer. $N$ Engl J Med. 2011;364(19): 1817-1825.

2. Kanai M, Yoshimura K, Asada M, et al. A phase I/II study of gemcitabine-based chemotherapy plus curcumin for patients with gemcitabine-resistant pancreatic cancer. Cancer Chemother Pharmacol. 2011;68(1):157-164.

3. Oh SY, Kim HJ, Kim TH, et al. Pilot study of irinotecan/oxalipaltin (IROX) combination chemotherapy for patients with gemcitabine- and 5-fluorouracil-refractory pancreatic cancer. Invest New Drugs. 2010; 28(3):343-349.
4. Rothenberg ML, Moore MJ, Cripps MC, et al. A phase II trial of gemcitabine in patients with 5-FU-refractory pancreas cancer. Ann Oncol. 1996;7(4):347-353.

5. Cheon DJ, Orsulic S. Mouse models of cancer. Annu Rev Pathol. 2011;6:95-119.

6. Garber K. Realistic rodents? Debate grows over new mouse models of cancer. J Natl Cancer Inst. 2006;98(17):1176-1178.

7. Hoffman RM. Orthotopic is orthodox: why are orthotopic-transplant metastatic models different from all other models? J Cell Biochem. 1994;56(1):1-3.

8. Killion JJ, Radinsky R, Fidler IJ. Orthotopic models are necessary to predict therapy of transplantable tumors in mice. Cancer Metastasis Rev. 1998;17(3):279-284.

9. Richmond A, Su Y. Mouse xenograft models vs GEM models for human cancer therapeutics. Dis Model Mech. 2008;1(2-3):78-82.

10. Dorado J, Lonardo E, Miranda-Lorenzo I, Heeschen C. Pancreatic cancer stem cells: new insights and perspectives. J Gastroenterol. 2011;46(8):966-973.

11. Hermann PC, Huber SL, Herrler T, et al. Distinct populations of cancer stem cells determine tumor growth and metastatic activity in human pancreatic cancer. Cell Stem Cell. 2007;1(3):313-323.

12. Jordan CT, Guzman ML, Noble M. Cancer stem cells. $N$ Engl J Med. 2006;355(12):1253-1261.

13. Lee CJ, Dosch J, Simeone DM. Pancreatic cancer stem cells. J Clin Oncol. 2008;26(17):2806-2812.

14. Reya T, Morrison SJ, Clarke MF, Weissman IL. Stem cells, cancer, and cancer stem cells. Nature. 2001;414(6859):105-111.

15. Visvader JE, Lindeman GJ. Cancer stem cells in solid tumours: accumulating evidence and unresolved questions. Nat Rev Cancer. 2008;8(10):755-768.

16. Hutmacher DW. Biomaterials offer cancer research the third dimension. Nat Mater. 2010;9(2):90-93.

17. Hutmacher DW, Horch RE, Loessner D, et al. Translating tissue engineering technology platforms into cancer research. J Cell Mol Med. 2009;13(8A):1417-1427.

18. Kim JB. Three-dimensional tissue culture models in cancer biology. Semin Cancer Biol. 2005;15(5):365-377.

19. Lee J, Cuddihy MJ, Kotov NA. Three-dimensional cell culture matrices: state of the art. Tissue Eng Part B Rev. 2008;14(1):61-86.

20. Lutolf MP, Hubbell JA. Synthetic biomaterials as instructive extracellular microenvironments for morphogenesis in tissue engineering. Nat Biotechnol. 2005;23(1):47-55.

21. Thomas V, Zhang X, Catledge SA, Vohra YK. Functionally graded electrospun scaffolds with tunable mechanical properties for vascular tissue regeneration. Biomed Mater. 2007;2(4):224-232.

22. Thomas V, Zhang X, Vohra YK. A biomimetic tubular scaffold with spatially designed nanofibers of protein/PDS bio-blends. Biotechnol Bioeng. 2009;104(5):1025-1033.

23. Zhang X, Thomas V, Vohra YK. In vitro biodegradation of designed tubular scaffolds of electrospun protein/polyglyconate blend fibers. J Biomed Mater Res A. 2009;89(1):135-147.

24. Zhang X, Thomas V, Vohra YK. Two ply tubular scaffolds comprised of proteins/poliglecaprone/polycaprolactone fibers. J Mater Sci. 2010;21(2):541-549.

25. Zhang X, Thomas V, Xu Y, Bellis SL, Vohra YK. An in vitro regenerated functional human endothelium on a nanofibrous electrospun scaffold. Biomaterials. 2010;31(15):4376-4381.

26. Zhang X, Xu Y, Thomas V, Bellis SL, Vohra YK. Engineering an antiplatelet adhesion layer on an electrospun scaffold using porcine endothelial progenitor cells. J Biomed Mater Res. 2011;97(2): 145-151.

27. Gaviraghi M, Tunici P, Valensin S, et al. Pancreatic cancer spheres are more than just aggregates of stem marker-positive cells. Biosci Rep. 2011;31(1):45-55.

28. Jimeno A, Feldmann G, Suarez-Gauthier A, et al. A direct pancreatic cancer xenograft model as a platform for cancer stem cell therapeutic development. Mol Cancer Ther. 2009;8(2):310-314. 
29. Louvet C, Labianca R, Hammel P, et al. Gemcitabine in combination with oxaliplatin compared with gemcitabine alone in locally advanced or metastatic pancreatic cancer: results of a GERCOR and GISCAD phase III trial. J Clin Oncol. 2005;23(15):3509-3516.

30. Dvir T, Timko BP, Kohane DS, Langer R. Nanotechnological strategies for engineering complex tissues. Nat Nanotechnol. 2011;6(1):13-22.

31. Balasundaram G, Webster TJ. An overview of nano-polymers for orthopedic applications. Macromol Biosci. 2007;7(5):635-642.

32. Weiler A, Hoffmann RF, Stahelin AC, Helling HJ, Sudkamp NP. Biodegradable implants in sports medicine: the biological base. Arthroscopy. 2000;16(3):305-321.

33. Fischbach C, Chen R, Matsumoto T, et al. Engineering tumors with 3D scaffolds. Nat Methods. 2007;4(10):855-860.
34. Hong SP, Wen J, Bang S, Park S, Song SY. CD44-positive cells are responsible for gemcitabine resistance in pancreatic cancer cells. Int J Cancer. 2009;125(10):2323-2331.

35. Weber GF, Bronson RT, Ilagan J, Cantor H, Schmits R, Mak TW. Absence of the CD44 gene prevents sarcoma metastasis. Cancer Res. 2002;62(8):2281-2286.

36. Zhu J, He J, Liu Y, Simeone DM, Lubman DM. Identification of glycoprotein markers for pancreatic cancer CD24+CD44+ stem-like cells using nano-LC-MS/MS and tissue microarray. J Proteome Res. 2012;11(4):2272-2281.

37. Sergeant G, Vankelecom H, Gremeaux L, Topal B. Role of cancer stem cells in pancreatic ductal adenocarcinoma. Nat Rev Clin Oncol. 2009;6(10):580-586.
International Journal of Nanomedicine

\section{Publish your work in this journal}

The International Journal of Nanomedicine is an international, peerreviewed journal focusing on the application of nanotechnology in diagnostics, therapeutics, and drug delivery systems throughout the biomedical field. This journal is indexed on PubMed Central, MedLine, CAS, SciSearch $\AA$, Current Contents ${ }^{\circledR} /$ Clinical Medicine,

\section{Dovepress}

Journal Citation Reports/Science Edition, EMBase, Scopus and the Elsevier Bibliographic databases. The manuscript management system is completely online and includes a very quick and fair peer-review system, which is all easy to use. Visit http://www.dovepress.com/ testimonials.php to read real quotes from published authors. 